\title{
Delirium in the acute hospital setting: the role of psychiatry
}

\author{
James M. FitzGerald (1) \& Annabel Price
}

\begin{abstract}
SUMMARY
In this overview we discuss the role of psychiatry in managing delirium in acute hospital admissions. We briefly discuss the role psychiatry can offer in four main domains: (a) assessment; (b) management; (c) recovery; and (d) paradigm, education and research. In the assessment section we discuss accurately detecting delirium in the context of comorbid mixed neuropsychiatric syndromes, including depression and dementia, and the clinical importance of delirium subtyping. The management section briefly outlines pharmacological and nonpharmacological approaches to delirium and their evidence-based rationale. The recovery section focuses on the effect delirium can have on cognitive decline, mental health and long-term health, including functional outcome and need for institutional care after hospital discharge. Finally, we outline the role of psychiatry in delirium research and education. We hope that this article will encourage clinicians to reflect on their current practice and consider holistic and evidence-based care for this vulnerable population in the acute hospital setting.
\end{abstract}

\section{LEARNING OBJECTIVES}

After reading this article you will be able to:

- understand the epidemiology and prognosis of delirium in the acute hospital setting

- recognise that delirium often occurs in mixed presentations, which may require detailed longitudinal phenomenological profiling

- appreciate the current evidence-based approaches to delirium care in the acute hospital setting

\section{KEYWORDS}

Organic syndromes; antipsychotics; dementia; phenomenology; delirium.

Delirium is a major public health concern internationally; and as the biopsychosocial syndrome par excellence, its management can serve as an index of the quality of care an older patient receives in hospital (Jackson 2017). Delirium is often defined as an acute-onset neuropsychiatric syndrome, marked by changes to cognitive domains (e.g. attention and memory) and wider behavioural changes (e.g. sleepwake cycle and motor disturbances), occurring in the context of medical illness (Inouye 2014).
Delirium is a complex syndrome that has a highly heterogeneous and fluctuating phenomenological profile, which results in a wide differential diagnosis. The aetiology is multifactorial and includes both modifiable (e.g. acute critical illness) and non-modifiable (e.g. advanced age) risk factors (Vasilevskis 2012). In the acute hospital setting, it has a prevalence of $20 \%$ among general medical admissions, and this increases to over $50 \%$ in medical admissions of older people (Davis 2013; Inouye 2014). In more specialist clinical settings such as the intensive care unit (ICU) and palliative care, its prevalence can reach over 80\% (Krewulak 2018; Hosie 2013). It is associated with a variety of detrimental clinical outcomes, including increased length of hospital stay and increased risk of morbidity, mortality and dementia (Inouye 2014). Delirium is also a distressing experience for both patients and families. Indeed, qualitative research has reported on the experiential distress and resulting features of anxiety, depression and post-traumatic stress-like features that patients have following an in-patient episode of delirium (Grover 2014; Martins 2016). Families and caregivers are also affected by the presence of delirium and it can increase burnout and carer distress (Finucane 2017). Following discharge, patients often face long-term consequences such as reduced adaptive functioning and an increased risk of needing institutional care (Jackson 2016). Despite all of these factors, delirium remains clinically underdetected and hence poorly managed in up to two-thirds of cases (Fong 2017). Psychiatrists' skills in assessing complex psychopathology, coupled with specialist knowledge of pharmacological and non-pharmacological interventions, enable them to provide the necessary support required to help improve detection, optimise management and support recovery.

\section{Assessment}

\section{Delirium phenomenology and diagnosis}

At present there is no gold-standard biochemical test to accurately detect delirium (Toft 2019). Neuroimaging has a limited role clinically in delirium assessment, with current research highlighting the non-specific findings such as cerebral atrophy and neural dysconnectivity (Nitchingham 2018). One study reported positive findings in only $14.5 \%$
James FitzGerald is a trainee psychiatrist working in the Cambridgeshire and Peterborough NHS Foundation Trust, and a National Institute for Health Research (NIHR) Academic Clinical Fellow in the Department of Psychiatry at the University of Cambridge, UK. His research interests include delirium, dementia and psychodynamic psychiatry. Annabel Price is a consultant in liaison psychiatry for older adults working in Addenbrooke's Hospital, Cambridge. She is also the Associate Specialist Director for Palliative Care, Cambridge Institute of Public Health (University of Cambridge), and Vice Chair of the Royal College of Psychiatrists' Faculty of Liaison Psychiatry, London, UK. Correspondence Dr James FitzGerald. Email: james.fitzgerald@ cpft.nhs.uk

First received 29 Jan 2020 Final revision 16 May 2020 Accepted 11 Jun 2020

\section{Copyright and usage}

(c) The Authors 2020. Published by Cambridge University Press on behalf of the Royal College of Psychiatrists. This is an Open Access article, distributed under the terms of the Creative Commons Attribution licence (http://creativecommons.org/ licenses/by/4.0/), which permits unrestricted re-use, distribution, and reproduction in any medium, provided the original work is properly cited. 
of patients undergoing structural (magnetic resonance imaging and computed tomography) neuroimaging investigations (Hijazi 2018). Therefore, in the absence of reliable biomarkers, the detection and assessment of delirium is informed largely by a review of the patient's clinical psychopathology/ phenomenology.

Although there are an estimated 340 synonyms for delirium. depending on the clinical setting, the consensus from the DSM-5 and ICD-10 classification systems recognises all acute disturbances of global cognitive functioning as delirium (World Health Organization 1992; American Psychiatric Association 2013). The recently available ICD-11 continues with this presentation of delirium as a clinical entity (World Health Organization 2019).

Delirium is a complex syndrome reflective of generalised neural dysfunction and yet lacks a pathognomonic feature. Accumulated research into the phenomenology of delirium indicates that there are four main domains of disturbed phenomenology: circadian integrity, executive cognition, orders of consciousness, and temporality (Fig. 1) (Hobson 2011; Franco 2013; Leonard 2015). Circadian integrity is composed of two subdomains, motor behaviour and disturbances in the sleep-wake cycle (Fitzgerald 2013). Executive cognition refers to such cognitive processes as attention, memory, language and orientation (Lindroth 2019; Mitchell 2018). The third domain, the orders of consciousness, are the functional modes by which executive cognition is enabled. They are divided into primary and secondary consciousness, but are experienced as an integrated whole. Primary consciousness refers to sensory and perceptual experience, and secondary consciousness encompasses metacognition, i.e. awareness of one's own thought processes. These aspects of consciousness serve as the modalities by which delirium, waking and sleep consciousness possess a phenomenological continuum (Hobson 2011). The fourth dimension is temporality and results from the convergence of the neurocognitive reserve of the individual and the influence of the pathological processes on this reserve (Cunningham 2013). This domain is critical to detecting delirium in the acute hospital setting, given the plethora of research highlighting the heterogeneity in the temporal duration of delirium features (Kim 2018). It is clinically essential to recognise the temporal pattern of delirium phenomenology. Delirium has an acute onset of features, with a prodromal change in mental state lasting an estimated 2-3 days and including lethargy, malaise and restlessness. A fluctuating course is driven by disturbed circadian regulation, with features worse at night, lasting days to weeks in most cases (Trzepacz 2017). Taken together, an

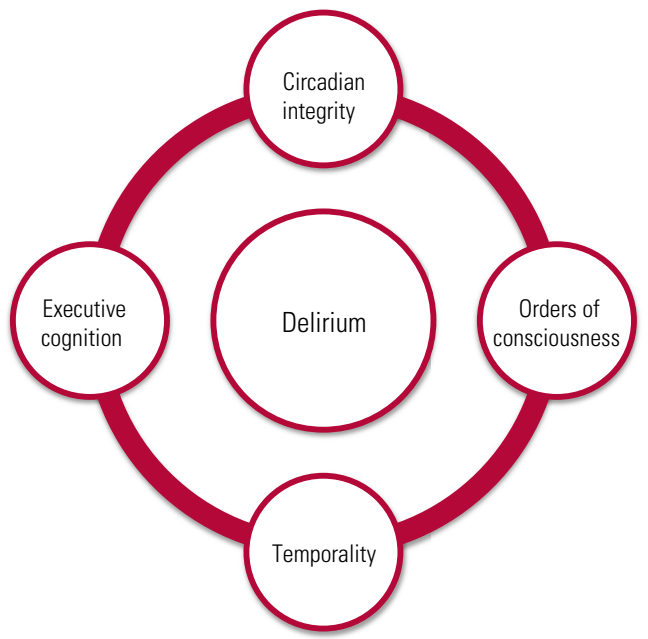

FIG 1

Multidimensional model of delirium phenomenology.

understanding of these domains is clinically relevant to the accurate psychiatric assessment of delirium, which includes both a detailed longitudinal analysis of its phenomenology and a focus on the temporal pattern of features and their severity (Adamis 2010).

\section{Subsyndromal delirium}

Subsyndromal delirium is a state characterised by the presence of delirium symptoms, but without the criteria for full syndromal delirium. It is associated with outcomes that are intermediate between full syndromal delirium and no delirium (Dosa 2007; Cole 2013). Although it is thought to include many features of full syndromal delirium, such as disturbances in motor behaviour, consciousness and sleep-wake rhythmicity, a comprehensive account has been impeded by the lack of clear diagnostic criteria (Boettger 2018). Although the frequency of full syndromal delirium has been well described, the frequency of subsyndromal delirium is less well defined and is estimated to occur in approximately $7-50 \%$ of older adult in-patients. This wide range is likely to be due to the clinical population studied and definition applied (Ouimet 2007; Bond 2012).

\section{Overlapping phenomenology and complex presentations}

The diagnosis and assessment of delirium is complicated by a wide differential, with depression and dementia being especially relevant. Delirium often exists in the context of neurocognitive disorders, with the prevalence of delirium superimposed on dementia reported at between 20 and 80\% (Fong 2017). Delirium superimposed on dementia is associated with a significantly higher risk of mortality, the need for long-term institutional care and 
functional and cognitive decline compared with delirium alone (Avelino-Silva 2017).

Although delirium is typically a transient disorder, it is increasingly recognised that delirium is often marked by incomplete recovery, with an estimated $20 \%$ of patients having persistent delirium that may last months (Cole 2008). In addition, dementia with Lewy bodies (DLB) is often described as the delirium-dementia continuum owing to the presence of shared features such as fluctuating cognition, visual hallucinations and disturbances in the sleep-wake cycle (Fong 2017). In a retrospective case-note study of patients undergoing review of dementia diagnosis at a tertiary referral unit, it was found that delirium was more closely associated with DLB than with Alzheimer's disease (Vardy 2014). A more recent study using electronic health records highlights the significantly higher occurrence of delirium in DLB than in Alzheimer's disease (FitzGerald 2019). There is unfortunately a lack of consensus regarding the differentiation between delirium and dementia, which has an impact on patient care and clinical outcomes (Richardson 2016). In the acute hospital setting, patients with complex neurocognitive features may be misdiagnosed and inappropriately treated with antipsychotics, which may lead to detrimental outcomes, given the high rate of neuroleptic sensitivity among people with DLB (Mueller 2017). Beyond the existence of dementia, older patients may present with mixed neuropsychiatric syndromes, including symptoms associated with depression, schizophrenia and mania. Given the non-specific manifestation of delirium phenomenology, specialist review by a psychiatrist is warranted to optimise patient care and recovery.

\section{Delirium subtypes}

Delirium can be categorised by clinically defined subtypes, with current evidence favouring the designation of subtypes based on motor activity profiles. There are currently four categories of motor subtype recognised: hyperactive, hypoactive, mixed and none (FitzGerald 2018). It has been reported that clinical motor subtypes of delirium differ in several ways, including detection rates, treatment experience, pathophysiology, duration of delirium episode and clinical outcome. Hypoactivity has been associated with metabolic causes and organ failure, whereas hyperactivity is more connected to substance-related delirium such as delirium tremens (Morandi 2017). Unfortunately, such studies have been found to have inconsistent findings due to heterogeneous methodology regarding motor subtype profiling (FitzGerald 2018). Despite these methodological limitations, hypoactive and mixed subtypes have been found to have a significantly poorer prognosis, with an estimated 1 in 3 patients dying during their hospital admission (Avelino-Silva 2018). Hypoactive motor profiles have been found to have higher associated mortality independent of factors such as comorbidity, age, delirium and severity of dementia (Kiely 2007). The association between hypoactive delirium and elevated mortality may be reflective of delayed detection of delirium, and hence more prolonged episodes (González 2009). Conversely, hyperactive delirium is associated with more frequent use of antipsychotics, higher detection rates and better outcomes (Meagher 2011).

\section{Methods to improve detection}

The underdetection of delirium is the biggest challenge to optimising delirium management and recovery (Ritter 2018). It has been reported that delirium is particularly underdetected in the emergency department and that approximately $77 \%$ of patients with delirium in the emergency department continue to have delirium during their hospital admission (Han 2017).

In clinical practice, assessing delirium requires a longitudinal perspective, which includes repeated assessments. Such a process can be informed by using validated tools to optimise the diagnostic ability of ward staff and provide reliable longitudinal assessments following review by a psychiatrist. There are an estimated 24 delirium detection tools available, and many have been translated into a variety of languages (Adamis 2010). In the acute hospital setting, both the Confusion Assessment Method (CAM) and the 4AT have been widely used as screening methods to help optimise delirium detection and monitor recovery. Although the 4AT is in widespread use in the UK's National Health Service (NHS) and has demonstrated high diagnostic performance, the CAM has been adapted and applied to a multitude of clinical settings, including the emergency department and ICU as well as numerous ward settings (Gélinas 2018; Shenkin 2019). Of the rapid bedside tests of attention and arousal, the Observational Scale of Level of Arousal (OSLA) and the Richmond AgitationSedation Scale (RASS) have demonstrated high sensitivity and specificity for detecting delirium in older medical in-patients (Quispel-Aggenbach 2018). At present there is no consensus regarding the optimum routine screening system for delirium or the best tool to use. These tools are dependent on the training of the individuals and begin to lose their utility when patients present with mixed neuropsychiatric conditions such as dementia and affective disorders. In these complex contexts, more detailed tools have been developed and validated 
to optimise detection, such as the Delirium Rating Scale-Revised-98 (Adamis 2010). This instrument detects the wide variety of presenting features of delirium, combined with severity scores for each item and global severity scores (Trzepacz 2001). Again, this approach requires extensive training. In the context of subsyndromal delirium, there are no validated assessment tools, and such a diagnosis is based on specialist review by a psychiatrist, who may apply an operationalised algorithm adapted from the literature (Trzepacz 2012). In the context of motor subtyping, several methods have been validated. These include the Delirium Motor Subtyping Scale (DMSS) and the abbreviated 4-item DMSS. Both these tools have been found to have high cross-sectional and longitudinal reliability (Fitzgerald 2016; Boettger 2017). This is clinically helpful as it may enable ward staff to accurately detect subtypes and monitor their features longitudinally. Informal screening is consistently reported to be insufficient to accurately detect delirium in routine clinical practice, whereas validated screening tools and integrated diagnostic algorithms have demonstrated reliability and feasibility across several acute hospital settings (Grossmann 2014; Maclullich 2019). In real-world clinical practice each case of delirium is unique. Ward staff can be helped by their liaison (general hospital) psychiatry service to apply a suitable method for the particular patient to enable the necessary longitudinal monitoring of patient progress and recovery.

\section{Management}

\section{Cognitive-friendly hospitals and policy development}

The priority in approaching delirium in the acute hospital setting should be to optimise the conditions for its prevention. Indeed, comprehensive geriatric assessment with a view to reducing any modifiable risks for delirium is essential. Addressing polypharmacy is a key priority, especially rationalising any anticholinergic medications that may reduce cognitive function. Another priority is to identify and treat underlying causes and contributors. Delirium does not exist in isolation, but rather in a complex interaction of ward processes and acute illnesses. To provide optimum care for the ageing population, the concept of the cognitive-friendly hospital has emerged. The characteristics that are required for such a hospital according to Maclullich et al (2013) are outlined in Box 1. In the NHS clinical context, the National Institute for Health and Care Excellence (NICE) (2010) offers guidelines at a national level (Clinical Guidelines CG103) that can be adapted to the individual hospital setting. These guidelines describe the current evidence in four key domains: (a) risk factor assessment; (b) interventions to prevent and manage delirium; (c) delirium screening and assessment; and (d) information, support and communication. Unfortunately, national and international evidence reports on the persistent gap between delirium policy guidelines such as NICE CG103 and their application through associated quality standards (such as NICE QS63; NICE 2014) (Lamond 2018).

Liaison psychiatry can offer a valuable contribution to local, regional and national policy development and hospital management structures with a view to improving care beyond the unit of the patient or ward. The Royal College of Psychiatrists has highlighted the role liaison psychiatry can offer by supporting ward staff to apply these guidelines in the real-world clinical setting (Royal College of Psychiatrists 2013, 2019a).

\section{Multicomponent interventions and liaison psychiatry multidisciplinary teams}

The current evidence recommends that multicomponent interventions are the first-line management and prevention strategy for delirium in the acute hospital setting. These interventions consist of nurse-led enhanced care plans that minimise and contain modifiable risk factors for delirium, such as dehydration, immobility, pain and malnutrition (Teale 2017; Oberai 2018). In addition to the liaison psychiatry service, the delivery of multicomponent interventions would be supported by other members of the multidisciplinary team such as physiotherapists (mobility recovery), dieticians (patient nutrition) and elder care physicians (comprehensive geriatric assessment).

In the largest and most up-to-date systematic review and meta-analysis on the subject, Hshieh et al (2018) appraise the impact and implementation of multicomponent interventions on delirium detection and management in the acute hospital setting. In total, 44 studies set in a wide variety of international (USA, Europe and South America) acute hospital settings were included in the systematic review and 14 of those were included in the metaanalysis. The authors concluded that the evidence

BOX 1 Key characteristics of cognitive-friendly hospitals

- Guidelines for the prevention and management of delirium

- Routine delirium screening

- Delirium education for frontline staff, and for patients and their families

- Specialist services

(Maclullich 2013) 
supported the implementation of multicomponent interventions in the acute hospital setting to reduce delirium incidence, the rate of patient falls, the length of hospital stay and the rate of transfer to long-term institutional care. Six of the included studies explored barriers and facilitators in implementing this approach to delirium care. The key facilitators included clinician leadership and changing organisational culture. However, the most prominent facilitator was the implementation of the policy measure to identify and empower an administrative champion (also known as a delirium champion). Ward-based nursing staff are in a key position to adopt this role, which could be supported by the liaison psychiatry service.

Given the prevalence and acute critical care needs of patients with delirium, nursing staff are in an important position to initiate multicomponent interventions and deliver routine delirium screening. However, neither NICE nor the Royal College of Nursing endorses any particular methods of implementing these interventions. International and national evidence suggests that, in the absence of routine patient screening or educational training for ward staff, patients with delirium may be missed and hence not treated appropriately (NICE 2014; Saczynski 2014; Yue 2014). In qualitative and quantitative studies on nurses' experience, perceptions and management of delirium, the most consistent barrier reported is the lack of detail on implementation of policy/guidelines regarding delirium detection and management. In particular, respondents reported a lack of guidance on how to apply key factors identified in the literature, which include: nursing screening and detection of delirium; training and education for nurses; and guidelines on management of delirium in the acute hospital setting (Fan 2012; Awad 2019). Therefore, liaison psychiatry service input focused on these domains may be a suitable approach to operationalising professional delirium guidelines and delivering optimum delirium care for patients in the acute hospital setting. This approach would also help coordinate patient care and provide additional support to the role of family members/caregivers.

Delirium care can also benefit from input from the wider liaison psychiatry multidisciplinary team and, in addition to the role doctors can offer, liaison psychiatry nurses have invaluable roles in areas such as detection of delirium, monitoring treatment efficacy, role-modelling good care, and giving prescribing advice. Such input can be further supported by the provision of high-quality mediation skills to help ward teams coordinate care with patients and families. Finally, the use of assessments under the Mental Capacity Act, Deprivation of Liberty Safeguards (DoLS) and occasionally the Mental
Health Act 1983 may be required to support management of patients with a delirium whose risks are significant. Such expertise can enable ward teams to support patients within the appropriate legal framework and protect patient rights.

\section{Pharmacotherapy}

Specialist knowledge of psychotropic medication is another key service that psychiatry can offer in the management of delirium. The current consensus regarding the pharmacological management of delirium proposes that psychotropic medication be used with caution and only for situations that have not yielded to non-pharmacological interventions (NICE 2010, 2014). Research has indicated that patients presenting with hyperactive delirium tend to be prescribed antipsychotics more frequently, likely owing to the distress and or the psychotic symptoms experienced (Meagher 2011). Conventionally, the antipsychotic of choice is haloperidol, given its absence of anticholinergic side-effects (Yoon 2013). The use of antipsychotics is controversial, and there is little evidence to indicate that they should be used to treat delirium directly (Neufeld 2016; Burry 2018). In congruence with this, there is no robust evidence to suggest that at-risk patients should be given antipsychotics prophylactically (Oh 2019).

Generally, antipsychotics are associated with a wide variety of adverse effects, such as prolonged QTc, torsades de pointes, and extrapyramidal and anticholinergic side-effects (Huhn 2019). In the context of delirium, antipsychotics have been found to be relatively safe if given for the duration of the detected delirium, for example 3-7 days. In a prospective observational study of 2453 acutely admitted patients who experienced an episode of delirium, it was found that approximately $1 \%$ experienced an adverse event attributable to antipsychotic use. Of note, the authors found no deaths attributable to antipsychotic use (Hatta 2014). However, caution must be used when DLB is suspected, particularly in the context of spontaneous Parkinsonism, rapid eye movement (REM) sleep behaviour disorder or visual hallucinations preceding acute cognitive decline (McKeith 2017). When required, antipsychotics should be prescribed for distress/agitation during the delirium episode where the patient may be a risk to themselves or others. They should be prescribed at the lowest effective dose for the shortest period and regularly reviewed for efficacy (NICE 2010, 2014). Psychiatry can offer additional advice and recommendations on the choice of antipsychotic if a patient's clinical profile requires a more careful consideration of its pharmacological properties, taking into consideration, for example, route of administration, comorbidities such as Parkinson's 
disease, cardiac history, and history of falls and hypotension. However, it is highly recommended that antipsychotics are reviewed prior to discharge and discontinued wherever possible, given the consistent finding that approximately a quarter of antipsychotics initiated for older patients in acute hospital settings continue after discharge (Herzig 2016; Loh 2014).

Finally, there is no evidence to support the use of benzodiazepines in managing delirium not associated with alcohol withdrawal (Lonergan 2009). There is limited emerging evidence to suggest that dexmedetomidine (an alpha-2 adrenergic agonist) may be a reasonable alternative to antipsychotics to manage agitation in ventilated ICU patients (Flükiger 2018). More recent studies have proposed that novel substances such as melatonin may have clinical utility due to its effect on sleep, but there is insufficient evidence to endorse this in the mainstream approach to delirium (Chen 2016).

\section{Models of delirium care}

As a psychiatry subspecialty, liaison psychiatry operates in the main via a 'referral-response' model, although models and composition of teams vary. This reactive (as opposed to proactive) model may risk missing opportunities for implementing preventive and early management strategies that may lead to improved outcomes for those at risk of delirium. The Royal College of Psychiatrists, for example, recommends that age-inclusive services have suitable embedded expertise to meet the specific needs of older people (Royal College of Psychiatrists 2019b). Interest is growing in more integrated models of liaison psychiatry, although the evidence base for clinical effectiveness and cost-effectiveness is in development. The ongoing HOME study based in the UK is a notable example of developing a more proactive approach to psychological medicine in the acute hospital setting (Walker 2019).

Although this article, and indeed the majority of literature on delirium, pertains to the acute hospital setting, it should be acknowledged that delirium occurs in a range of settings and therefore psychiatrists need to acquire skills in delirium management as a core aspect of their training. In addition to this consideration, integrated care models outside the typical hospital ward model are something to aspire to and have been implemented in some settings: a good example is the management and recovery approach to postoperative delirium (McDonald 2018).

\section{Recovery}

\section{The impact and experience of delirium}

Owing to its wide-ranging impact, recovery from delirium must be considered from different perspectives, which include cognition, physical health and mental health. Recovery is also informed by each patient's particular journey through the acute hospital setting. For example, patients presenting with delirium to the emergency department tend to have significantly longer hospital stays, higher 30-day mortality rates and higher 30-day readmission rates (Kennedy 2014). Such vulnerable patients have significantly higher rates of admission to the ICU and transfer to post-acute institutional care facilities on discharge from hospital (Han 2009; Kennedy 2014).

In the ICU setting, delirium is associated with an increased risk of mortality both during admission and following discharge. In this setting, delirium is associated with an increased ICU length of stay and patients are significantly more likely to undergo tracheostomy. It is also associated with prolonged mechanical ventilation time and total hospital length of stay (Mehta 2015). Post-operative delirium has been found to be associated with an increased rate of ICU stays that last more than 5 days, significantly higher rate of 30-day readmissions to acute hospital and higher rate of discharge to a permanent care home placement (Raats 2015). When patients are discharged to a postacute care facility following an episode of delirium it has been reported that they have significantly increased risk of mortality and higher 30-day acute hospital readmission rates (Kosar 2017). Taken together these clinical factors may have a detrimental impact on patient recovery in both cognitive and wider functional domains.

Not surprisingly, delirium is a distressing experience for patients, families, carers and healthcare staff (Grover 2014; Martins 2016). Qualitative research has reported on the experiential distress and resulting features of anxiety and depression that patients have after an in-patient episode of delirium (Whitehorne 2015). In particular, post-traumatic stress disorder (PTSD) is emerging as a recognised consequence of delirium and it has been suggested that it should be more routinely followed up, given its potential impact on patient recovery and quality of life (Teale 2013; Bolton 2019). However, many patients do not report their symptoms as they feel that the delirium experience is evidence of a severe mental illness associated with stigma (Kim 2017). As mentioned earlier, the presence of delirium can increase burnout and carer distress in families and caregivers (Finucane 2017). Patient recovery can be optimised, however, by engaging with orientation strategies and emotional support, which can significantly ameliorate the experiential distress of delirium (Halloway 2014). Consultation with liaison psychiatry services may offer advice regarding suitable support that patients 
and their families may require following discharge from hospital. However, given the growing literature highlighting the need for follow-up in the context of PTSD, mood and anxiety symptoms, service development to set up brief post-discharge clinical liaison clinics may be warranted.

\section{The interface between delirium and dementia}

Recovery from delirium is an ill-defined concept in the literature, and although it is widely considered that delirium is often reversible, accumulating evidence suggests that it is often marked by incomplete resolution (Witlox 2010; Adamis 2014). Indeed, it has been found that, for each day a patient has delirium in the emergency department, a significantly worse long-term cognitive and functional outcome has been reported (Han 2017). It has also been reported in a systematic review of 18 cohort studies that the proportions of patients with persistent delirium at point of discharge and at 1, 3 and 6 months are approximately 44.7\%, 32.8\%, 25.6\% and $21 \%$ respectively (Cole 2008). According to Inouye et al (2014), delirium can be a marker of the vulnerable brain, with a reduction in reserve capacity to withstand noxious insults. Therefore, delirium has a negative impact on the trajectory of normal cognitive ageing by adding a series of punctuated stages of decline and recovery associated with each episode of delirium (Fong 2017). These insults on cognitive function can consequently increase the risk in some vulnerable patients of developing dementia. This further reinforces the complex interface between delirium and dementia, and the postdischarge cognitive trajectory towards dementia that many patients with delirium may develop in the community (Fong 2015). To tackle this challenge, follow-up review of patients in the regional older people's mental health service (e.g. memory clinics) may help support patients and families to address the challenge and trajectory of cognitive impairment.

\section{Paradigm, education and research}

Good practice in the identification, assessment and management of delirium is a 'life skill' for medical professionals, especially those working with older people, but because the condition straddles the traditional separation of 'mind' and 'body' medicine it risks being neglected in more specialty-focused medical training programmes and therefore remaining underrecognised even in acute medical settings. Delirium may be most easily recognised when there is an obvious change in behaviour, psychiatric symptoms or difficulty in providing medical care. This may have contributed to a culture in which delirium is commonly conceptualised as a 'psychiatric' problem (albeit with an 'organic' underlying cause). An improvement in practice therefore requires a shift in culture whereby training in good delirium care is everybody's business and core to medical training throughout.

To optimise delirium detection, management and recovery in the acute hospital setting, further educational support and research approaches are warranted. Psychiatry as a discourse and as a practice can inform the multidisciplinary research required to improve the development of screening methods and routine profiling approaches across the clinical settings in which delirium is most manifest. At the centre of this issue is the role of phenomenology, which has often been cited as the rationale for the different approaches taken to manage delirium (Meagher 1998). Indeed, future pharmacotherapy trials may adapt their approach to targeting specific features of delirium, namely positive psychotic symptoms or perhaps clinical subtypes (e.g. hypoactive versus hyperactive) to yield more innovative findings. Moreover, the complex interface between delirium and dementia requires further investigation with a view to creating more reliable methods of either differentiating between these two neurocognitive syndromes, or identifying methods that can measure the impact of delirium on the vulnerable brain. In particular, neuroimaging combined with detailed phenomenological profiling may yield such benefits, particularly in elucidating the interface between delirium and DLB.

Gaps in knowledge and training have consistently been cited as critical barriers to implementing best practice care for people with delirium. Knowledge pertaining to screening, risk factor detection and reduction, and management of distress/psychosis are often cited as recurrent themes. Congruent with this, the 2011 National Audit of Dementia in acute hospitals in England and Wales found that only one-third of staff felt they had received adequate training or guidance in dementia care (Royal College of Psychiatrists 2012). In addition, ward teams may be uncertain about the optimal approach to engage with the specific processes of discharge planning, specialist out-patient referrals, older adult mental health requirements, and more intimate discussions with patients and families regarding the experience and impact of delirium. Liaison psychiatry services are well positioned to work collaboratively with ward teams to deliver the quality and provision of such information and skills. However, it is important to reiterate the importance of liaison psychiatry services delivering specific expertise in managing the needs of older people as compared with those of the younger adult population. This has been highlighted by the Royal College of Psychiatrists in a recent position statement (Royal 
MCO answers
College of Psychiatrists 2019a). Such an approach may enable the ward team to enhance its capacity to implement best-practice guidelines when engaging with patients with delirium and to improve the general approach with this vulnerable patient cohort.

\section{Discussion}

Delirium is an acute-onset neuropsychiatric emergency that presents across multiple acute clinical care settings. This article has outlined the different forms of support psychiatry can offer (Fig. 2). Psychiatry teams can provide assessment in the context of complex neuropsychiatric phenomenology and its impact on patients' mental health. Moreover, the liaison psychiatry service can work collaboratively with the ward team to optimise patient management and monitor its efficacy. Psychotherapeutic and general mediation skills can help support ward teams, families, carers and patients to aid in recovery from delirium. Old age psychiatrists are particularly skilled in dealing with the impact of delirium on cognitive functioning and advanced dementia. Indeed, the versatility of contemporary older people's mental health services enables psychiatry to offer a coherent and invaluable approach to delirium across domains.

Delirium is not a condition that exists in isolation and it is often the outcome of the general approach to care of the older patient in the acute hospital setting. Hence, a more multifaceted approach may have a

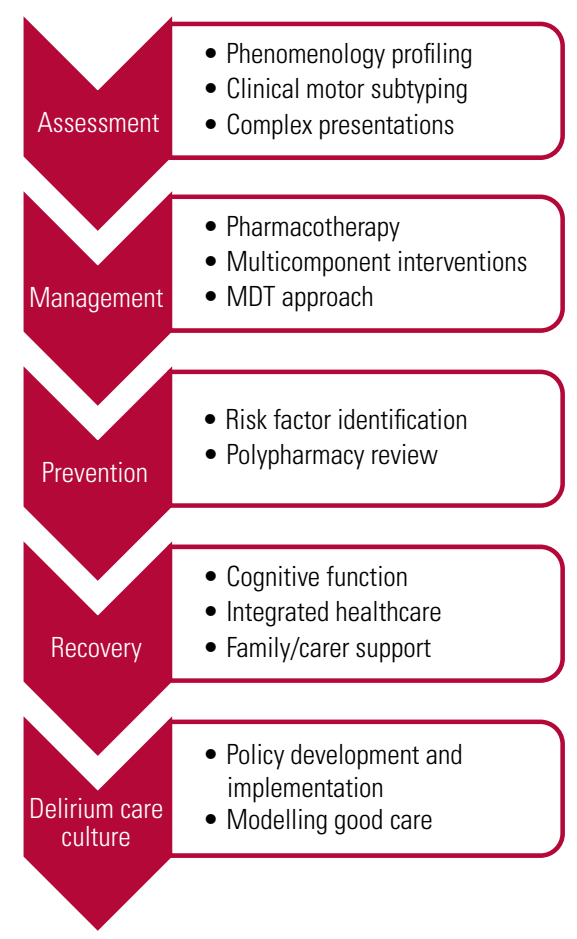

FIG 2 Psychiatry support for the multidisciplinary team (MDT). positive impact on delirium prevention, detection and management. Therefore, robust policy and practice initiatives are required to reconstruct and adapt the hospital setting to address the acute care needs of the ageing population across various clinical settings. Psychiatry is in a key position to support the optimisation of care regarding delirium in the acute hospital setting and provide a meaningful contribution to the evolving elder care discourse. Although perhaps as important as any of the crucial clinical contributions that psychiatry can offer would be a change in perspective so that delirium is regarded as an index of the quality of care that patients can receive in the acute hospital setting.

\section{Acknowledgements}

We thank Professor John O’Brien for reviewing an early draft of this article.

\section{Author contributions}

The authors contributed equally to the conception, writing and final submission of this article.

\section{Declaration of interest}

None.

ICMJE forms are in the supplementary material, available online at https://doi.org/10.1192/bja. 2020.44

\section{References}

Adamis D, Sharma N, Whelan PJ, et al (2010) Delirium scales: a review of current evidence. Aging \& Mental Health, 14: 543-55.

Adamis D, Devaney A, Shanahan E, et al (2014) Defining 'recovery' for delirium research: a systematic review. Age and Ageing, 44: 318-21.

American Psychiatric Association (2013) Diagnostic and Statistical Manual of Mental Disorders (5th edn) (DSM-5). American Psychiatric Publishing.

Avelino-Silva TJ, Campora F, Curiati JA, et al (2017) Association between delirium superimposed on dementia and mortality in hospitalized older adults: a prospective cohort study. PLoS Medicine, 14(3): e1002264.

Avelino-Silva TJ, Campora F, Curiati JAE, et al (2018) Prognostic effects of delirium motor subtypes in hospitalized older adults: a prospective cohort study. PloS One, 13(1): e0191092.

Awad SA (2019) Critical care nurses' knowledge, perception and barriers regarding delirium in adult critical care units. American Journal of Nursing, 7: 193-8.

Boettger S, Nuñez DG, Meyer R, et al (2017) Brief assessment of delirium subtypes: psychometric evaluation of the Delirium Motor Subtype Scale (DMSS)-4 in the intensive care setting. Palliative \& Supportive Care, 15: 535-43.

Boettger S, Nuñez DG, Meyer R, et al (2018) Subsyndromal delirium in the intensive care setting: phenomenological characteristics and discrimination of subsyndromal delirium versus no and full-syndromal delirium. Palliative \& Supportive Care, 16: 3-13.

Bolton C, Thilges S, Lane C, et al (2019) Post-traumatic stress disorder following acute delirium. Journal of Clinical Psychology in Medical Settings [Epub ahead of print] 10 Dec. Available from https://www.springer.com/ journal/10880 
Bond SM, Dietrich MS, Shuster Jr JL, et al (2012) Delirium in patients with head and neck cancer in the outpatient treatment setting. Supportive Care in Cancer, 20: 1023-30.

Burry L, Mehta S. Perreault MM, et al (2018) Antipsychotics for treatment of delirium in hospitalised non-ICU patients. Cochrane Database of Systematic Reviews, 66(6): CD005594.

Chen S, Shi L, Liang F, et al (2016) Exogenous melatonin for delirium prevention: a meta-analysis of randomized controlled trials. Molecular Neurobiology, 53: 4046-53

Cole MG, Ciampi A, Belzile E, et al (2008) Persistent delirium in older hospital patients: a systematic review of frequency and prognosis. Age and Ageing, 38: 19-26.

Cole MG, Ciampi A, Belzile E, et al (2013) Subsyndromal delirium in older people: a systematic review of frequency, risk factors, course and outcomes. Focus, 11: 534-43.

Cunningham C, Maclullich AM (2013) At the extreme end of the psychoneuroimmunological spectrum: delirium as a maladaptive sickness behaviour response. Brain, Behavior, and Immunity, 28: 1-13.

Davis DH, Kreisel SH, Terrera GM, et al (2013) The epidemiology of delirium: challenges and opportunities for population studies. American Journal of Geriatric Psychiatry, 21: 1173-89.

Dosa D, Intrator 0, McNicoll L, et al (2007) Preliminary derivation of a nursing home confusion assessment method based on data from the minimum data set. Journal of the American Geriatrics Society, 55: 1099-105.

Fan Y, Guo Y, Li Q, et al (2012) A review: nursing of intensive care unit delirium. Journal of Neuroscience Nursing, 44: 307-16.

Finucane AM, Lugton J, Kennedy C, et al (2017) The experiences of caregivers of patients with delirium, and their role in its management in palliative care settings: an integrative literature review. Psycho-Oncology, 26: $291-300$.

Fitzgerald JM, Adamis D, Trzepacz PT, et al (2013) Delirium: a disturbance of circadian integrity? Medical Hypotheses, 81: 568-76.

Fitzgerald J, O'Regan N, Adamis D, et al (2016) Concordance between the delirium motor subtyping scale (DMSS) and the abbreviated version (DMSS-4) over longitudinal assessment in elderly medical inpatients. International Psychogeriatrics, 28: 845-51.

FitzGerald JM (2018) Delirium clinical motor subtypes: a narrative review of the literature and insights from neurobiology. Aging \& Mental Health, 22: $431-43$.

FitzGerald JM, Perera G, Chang-Tave A, et al (2019) The incidence of recorded delirium episodes before and after dementia diagnosis: differences between dementia with Lewy bodies and Alzheimer's disease. Journal of the American Medical Directors Association, 20: 604-9.

Flükiger J, Hollinger A, Speich B, et al (2018) Dexmedetomidine in prevention and treatment of postoperative and intensive care unit delirium: a systematic review and meta-analysis. Annals of Intensive Care, 8(1): 92.

Fong TG, Davis D, Growdon ME, et al (2015) The interface between delirium and dementia in elderly adults. Lancet Neurology, 14: 823-32.

Fong TG, Inouye SK, Jones RN (2017) Delirium, dementia, and decline. JAMA Psychiatry, 74: 212-3.

Franco JG, Trzepacz PT, Meagher DJ, et al (2013) Three core domains of delirium validated using exploratory and confirmatory factor analyses. Psychosomatics, 54: 227-38.

Gélinas C, Bérubé M, Chevrier A, et al (2018) Delirium assessment tools for use in critically ill adults: a psychometric analysis and systematic review. Critical Care Nurse, 38: 38-49.

González M, Martínez G, Calderón J, et al (2009) Impact of delirium on short-term mortality in elderly inpatients: a prospective cohort study. Psychosomatics, 50: 234-8.

Grossmann FF, Hasemann W, Graber A, et al (2014) Screening, detection and management of delirium in the emergency department: a pilot study on the feasibility of a new algorithm for use in older emergency department patients: the modified Confusion Assessment Method for the Emergency Department (mCAM-ED). Scandinavian Journal of Trauma, Resuscitation and Emergency Medicine, 22(1): 19.
Grover S, Ghosh A Ghormode D (2014) Experience in delirium: is it distressing? Journal of Neuropsychiatry and Clinical Neurosciences, 27: 139-46.

Halloway S (2014) A family approach to delirium: a review of the literature. Aging \& Mental Health, 18: 129-39.

Han JH, Zimmerman EE, Cutler N, et al (2009) Delirium in older emergency department patients: recognition, risk factors, and psychomotor subtypes. Academic Emergency Medicine, 16: 193-200.

Han JH, Vasilevskis EE, Chandrasekhar R, et al (2017) Delirium in the Emergency Department and Its Extension into Hospitalization (DELINEATE) Study: effect on 6-month function and cognition. Journal of the American Geriatrics Society, 65: 1333-8.

Hatta K, Kishi Y, Wada K, et al (2014) Antipsychotics for delirium in the general hospital setting in consecutive 2453 inpatients: a prospective observational study. International Journal of Geriatric Psychiatry, 29: 253-62.

Herzig SJ, Rothberg MB, Guess JR, et al (2016) Antipsychotic use in hospitalized adults: rates, indications, and predictors. Journal of the American Geriatrics Society, 64: 299-305.

Hijazi Z, Lange P, Watson R, et al (2018) The use of cerebral imaging for investigating delirium aetiology. European Journal of Internal Medicine, 52: $35-9$.

Hobson A, Voss U (2011) A mind to go out of: reflections on primary and secondary consciousness. Consciousness and Cognition, 20: 993-7.

Hosie A, Davidson PM, Agar M, et al (2013) Delirium prevalence, incidence, and implications for screening in specialist palliative care inpatient settings: a systematic review. Palliative Medicine, 27: 486-98.

Hshieh TT, Yang T, Gartaganis SL, et al (2018) Hospital elder life program: systematic review and meta-analysis of effectiveness. American Journal of Geriatric Psychiatry, 26: 1015-33.

Huhn M, Nikolakopoulou A, Schneider-Thoma J, et al (2019) Comparative efficacy and tolerability of 32 oral antipsychotics for the acute treatment of adults with multi-episode schizophrenia: a systematic review and network meta-analysis. Lancet, 394: 939-51.

Inouye SK, Westendorp RG, Saczynski JS (2014) Delirium in elderly people. Lancet, 383: 911-22

Jackson TA, Wilson D, Richardson S, et al (2016) Predicting outcome in older hospital patients with delirium: a systematic literature review. International Journal of Geriatric Psychiatry, 31: 392-9.

Jackson TA, Gladman JR, Harwood RH, et al (2017) Challenges and opportunities in understanding dementia and delirium in the acute hospital. PLoS Medicine, 14(3): e1002247.

Kennedy M, Enander RA, Tadiri SP, et al (2014) Delirium risk prediction, healthcare use and mortality of elderly adults in the emergency department. Journal of the American Geriatrics Society, 62: 462-9

Kiely DK, Jones RN, Bergmann MA, et al (2007) Association between psychomotor activity delirium subtypes and mortality among newly admitted postacute facility patients. Journal of Gerontology Series A, 62: $174-9$

Kim SY, Kim JM, Kim SW, et al (2017) Perceived stigma and quality of life in patients following recovery from delirium. Journal of Clinical Psychiatry, 78: e744-9.

Kim SY, Kim JM, Kim SW, et al (2018) Do the phenotypes of symptom fluctuation differ among motor subtypes in patients with delirium? Journal of Pain and Symptom Management, 56: 667-77.

Kosar CM, Thomas KS, Inouye SK, et al (2017) Delirium during postacute nursing home admission and risk for adverse outcomes. Journal of the American Geriatrics Society, 65: 1470-5.

Krewulak KD, Stelfox HT, Leigh JP, et al (2018) Incidence and prevalence of delirium subtypes in an adult ICU: a systematic review and meta-analysis. Critical Care Medicine, 46(12): 2029-2035.

Lamond E, Murray S, Gibson CE (2018) Delirium screening in intensive care: a life saving opportunity. Intensive and Critical Care Nursing, 44: $105-9$.

leonard M, Adamis D, Saunders J, et al (2015) A longitudinal study of delirium phenomenology indicates widespread neural dysfunction. Palliative \& Supportive Care 13: 187-96. 
Lindroth H, Bratzke L, Twadell S, et al (2019) Predicting postoperative delirium severity in older adults: the role of surgical risk and executive function. International Journal of Geriatric Psychiatry, 34: 1018-28.

Loh KP, Ramdass S, Garb JL, et al (2014) From hospital to community: use of antipsychotics in hospitalized elders. Journal of Hospital Medicine, 9 : 802-4.

Lonergan E, Luxenberg J, Sastre AA (2009) Benzodiazepines for delirium. Cochrane Database of Systematic Reviews, 4: CD006379.

Maclullich AMJ, Anand A, Davis DH, et al (2013) New horizons in the pathogenesis, assessment and management of delirium. Age and Ageing, 42: 667-74.

Maclullich AMJ, Shenkin SD, Goodacre S, et al (2019) Implementing delirium screening. In The 4 'A's Test for Detecting Delirium in Acute Medical Patients: A Diagnostic Accuracy Study (Health Technology Assessment, no. 23.40). NIHR Journals Library.

Martins S, Fernandes L (2016) Delirium related distress in family: a nonsystematic review. European Psychiatry, 33: S218.

McDonald SR, Heflin MT, Whitson HE, et al (2018) Association of integrated care coordination with postsurgical outcomes in high-risk older adults: the Perioperative Optimization of Senior Health (POSH) initiative. JAMA Surgery, 153: 454-62.

McKeith IG, Boeve BF, Dickson DW, et al (2017) Diagnosis and management of dementia with Lewy bodies: fourth consensus report of the DLB Consortium. Neurology, 89: 88-100.

Meagher DJ, Trzepacz PT (1998) Delirium phenomenology illuminates pathophysiology, management, and course. Journal of Geriatric Psychiatry and Neurology, 11: 150-6.

Meagher DJ, Leonard M, Donnelly S, et al (2011) A longitudinal study of motor subtypes in delirium: relationship with other phenomenology, etiology, medication exposure and prognosis. Journal of Psychosomatic Research, 71: 395-403.

Mehta S, Cook D, Devlin JW, et al (2015) Prevalence, risk factors, and outcomes of delirium in mechanically ventilated adults. Critical Care Medicine, 43: 557-66.

Mitchell ML, Shum DH, Mihala G, et al (2018) Long-term cognitive impairment and delirium in intensive care: A prospective cohort study. Australian Critical Care, 31: 204-11.

Morandi A, Di Santo SG, Cherubini A, et al (2017) Clinical features associated with delirium motor subtypes in older inpatients: results of a multicenter study. American Journal of Geriatric Psychiatry, 25: 1064-71.

Mueller C, Ballard C, Corbett A, et al (2017) The prognosis of dementia with Lewy bodies. Lancet Neurology, 16: 390-8.

National Institute for Health and Care Excellence (2010) Delirium Diagnosis, Prevention and Management (Clinical Guideline CG103). NICE (http://www.nice.org.uk/CG103 [accessed 18 Dec 2019]).

National Institute for Health and Care Excellence (2014) Delirium Diagnosis, Prevention and Management (Quality Standards 0S103). NICE (http://www.nice.org.uk/00S103 [accessed 18 Dec 2019]).

Neufeld KJ, Yue J, Robinson TN, et al (2016) Antipsychotic medication for prevention and treatment of delirium in hospitalized adults: a systematic review and meta-analysis. Journal of the American Geriatrics Society, 64: 705-14.

Nitchingham A, Kumar V, Shenkin S, et al (2018) A systematic review of neuroimaging in delirium: predictors, correlates and consequences. International Journal of Geriatric Psychiatry, 33: 1458-78.

Oberai T, Laver K, Crotty M, et al (2018) Effectiveness of multicomponent interventions on incidence of delirium in hospitalized older patients with hip fracture: a systematic review. International Psychogeriatrics, 30: 481-92.

Oh ES, Needham DM, Nikooie R, et al (2019) Antipsychotics for preventing delirium in hospitalized adults. Annals of Internal Medicine, 171: 474-84.

Ouimet S, Riker R, Bergeon N, et al (2007) Subsyndromal delirium in the ICU: evidence for a disease spectrum. Intensive Care Medicine, 33: 1007-13.

Quispel-Aggenbach DWP, Holtman GA, Zwartjes HAHT, et al (2018) Attention, arousal and other rapid bedside screening instruments for delirium in older patients: a systematic review of test accuracy studies. Age and Ageing, 47: 644-53.
Raats JW, van Eijsden WA, Crolla RM, et al (2015) Risk factors and outcomes for postoperative delirium after major surgery in elderly patients. PloS One, 10(8): e0136071.

Richardson S, Teodorczuk A, Bellelli G, et al (2016) Delirium superimposed on dementia: a survey of delirium specialists shows a lack of consensus in clinical practice and research studies. International Psychogeriatrics, 28 : 853-61.

Ritter SR, Cardoso AF, Lins MM, et al (2018) Underdiagnosis of delirium in the elderly in acute care hospital settings: lessons not learned. Psychogeriatrics, 18: 268-75.

Royal College of Psychiatrists (2012) Report of the National Audit of Dementia Care in General Hospitals 2011. Healthcare Improvement Quality Partnership.

Royal College of Psychiatrists (2013) Liaison Psychiatry for Every Acute Hospital: Integrated Mental and Physical Healthcare (College Report CR183). Royal College of Psychiatrists.

Royal College of Psychiatrists (2019a) The Role of Liaison Psychiatry in Integrated Physical and Mental Healthcare (Position Statement PSO7) 19). Royal College of Psychiatrists.

Royal College of Psychiatrists (2019b) Position Statement on the Provision of Liaison Psychiatry Services across the Lifespan (Position Statement PS02/19). Royal College of Psychiatrists.

Saczynski JS, Kosar CM, Xu G, et al (2014) A tale of two methods: chart and interview methods for identifying delirium. Journal of the American Geriatrics Society, 62: 518-24.

Shenkin SD, Fox C, Godfrey M, et al (2019) Delirium detection in older acute medical inpatients: a multicentre prospective comparative diagnostic test accuracy study of the 4AT and the Confusion Assessment Method. BMC Medicine, 17(1): 138

Svenningsen $\mathrm{H}$ (2013) Associations between sedation, delirium and posttraumatic stress disorder and their impact on quality of life and memories following discharge from an intensive care unit. Danish Medical Journal, 60(4): B4630

Teale EA, Siddiqi N, Clegg A (2017) Non-pharmacological interventions for managing delirium in hospitalised patients. Cochrane Database of Systematic Reviews, 4: CD005995.

Toft K, Tontsch J, Abdelhamid S, et al (2019) Serum biomarkers of delirium in the elderly: a narrative review. Annals of Intensive Care, 9(1): 76

Trzepacz PT, Mittal D, Torres R, et al (2001) Validation of the Delirium Rating Scale-Revised-98: comparison with the delirium rating scale and the cognitive test for delirium. Journal of Neuropsychiatry and Clinical Neurosciences, 13: 229-42.

Trzepacz PT, Franco JG, Meagher DJ, et al (2012) Phenotype of subsyndromal delirium using pooled multicultural Delirium Rating Scale-Revised-98 data. Journal of Psychosomatic Research, 73: 10-17.

Trzepacz PT (2017) Delirium. In Neuro-Geriatrics: A Clinical Manual (eds B Tousi, J Cummings): 343-65. Springer.

Vardy E. Holt R, Gerhard A et al (2014) History of a suspected delirium is more common in dementia with Lewy bodies than Alzheimer's disease: a retrospective study. International Journal of Geriatric Psychiatry, 29: $178-81$

Vasilevskis EE، Han JH, Hughes CG, et al (2012) Epidemiology and risk factors for delirium across hospital settings. Best Practice \& Research Clinical Anaesthesiology, 26: 277-87.

Walker J, Burke K, Toynbee M, et al (2019) The HOME Study: study protocol for a randomised controlled trial comparing the addition of Proactive Psychological Medicine to usual care, with usual care alone, on the time spent in hospital by older acute hospital inpatients. Trials, 20(1): $1-9$

Whitehorne K, Gaudine A, Meadus R, et al (2015) Lived experience of the intensive care unit for patients who experienced delirium. American Journal of Critical Care, 24: 474-9.

Witlox J, Eurelings LS, de Jonghe JF, et al (2010) Delirium in elderly patients and the risk of postdischarge mortality, institutionalization, and dementia: a meta-analysis. JAMA, 304: 443-51. 
World Health Organization (1992) International Statistical Classification of Disease and Related Health Problems, Tenth Revision (ICD-10). WHO.

World Health Organization (2019) International Statistical Classification of Disease and Related Health Problems, Eleventh Revision (ICD-11). $\mathrm{WHO}$
Yoon HJ, Park KM, Choi WJ, et al (2013) Efficacy and safety of haloperidol versus atypical antipsychotic medications in the treatment of delirium. BMC Psychiatry, 13(1): 240

Yue J, Tabloski P, Dowal SL, et al (2014) NICE to HELP: operationalizing National Institute for Health and Care Excellence guidelines to improve clinical practice. Journal of the American Geriatrics Society, 62: 754-61.

\section{MCQs}

Select the single best option for each question stem

1 The estimated frequency of antipsychotic prescriptions initiated for older patients in acute hospital settings that continue after discharge is:
a $25 \%$
b $66 \%$
c $1 \%$
d $100 \%$
e $40 \%$

2 Of the following subtypes of delirium, the best clinical outcomes are for:

a hyperactive

b hypoactive

c hypoactive and mixed

d mixed

e hyperactive and mixed

\section{A recommended first-line treatment in} managing delirium is:

a an enhanced care plan

b single component interventions

c multicomponent interventions

d admission to a mental health ward

e watch and wait approach

4 As regards the pharmacotherapy of delirium:

a there is robust evidence to support the use of atypical over typical antipsychotics in managing delirium

b the use of antipsychotics is controversial and there is little evidence to indicate that they should be used to treat delirium directly

c typical antipsychotics such as haloperidol should be used prophylactically to reduce delirium

d risperidone is the antipsychotic of choice for managing delirium

e olanzapine is the antipsychotic of choice for managing delirium
5 The estimated proportion of patients with persistent delirium at 6 months post-discharge from hospital is:
a $41 \%$
b $81 \%$
c $21 \%$
d $31 \%$
e $11 \%$ 